\title{
Foundations for the future
}

\section{Challenges remain in understanding battery processes that govern operation and limit performance, but fundamental research holds the key for further development of beyond-lithium-ion technologies.}

Batteries store electricity in the form of chemical energy. Portable electronic devices, electrified transportation, and grid-scale applications require batteries that are environmentally benign, safe, possess high energy density and long cycle life, and consist of low-cost materials.

The commercialization of rechargeable lithium-ion batteries in the early 1990s is regarded as the most significant milestone in the revival of battery technologies since their invention by Alessandro Volta at the end of the 18th century. Despite remarkable achievements in the development of lithium-ion batteries in recent decades, the speed of battery development remains rather incremental ${ }^{1}$ : over the past 25 years the energy density of commercial lithium-ion batteries has increased fairly linearly by just under a factor of four ${ }^{2}$. State-of-the-art commercial lithium-ion batteries have an energy density of less than $300 \mathrm{Wh} \mathrm{kg}^{-1}$ (refs 3,4), which falls short of the US Department of Energy's target of $400 \mathrm{Wh} \mathrm{kg}^{-1}$ by 2017. While lithium-ion batteries will continue to be important for some years to come, a consensus has now formed that lithium-ion batteries will not be able to satisfy the energy storage requirements of the long-term future and new battery technologies are urgently needed ${ }^{5}$.

Batteries with new chemistries, often dubbed post lithium-ion batteries, have long been proposed to achieve higher energy density and longer cycle life. However, tremendous challenges remain in their development and many aspects of the underlying science of battery processes at electrodes, electrolytes and their interface are still poorly understood. Acquiring sufficient knowledge at the fundamental level is imperative to realizing the full potential of post lithium-ion batteries. In this issue, we present four post lithium-ion examples lithium-air, lithium-sulfur, lithium-metal and solid-state batteries - highlighting the understanding that has been acquired and the issues that still need solutions.

Lithium-air batteries use oxygen, which can be drawn from the air, as the active material at the positive electrode: upon discharge gaseous oxygen molecules gain electrons to form discharge products while upon charge the reverse reactions take place. The process may sound simple; however, the reactive reduced oxygen species can induce parasitic reactions with the electrolyte and positive electrode, causing problems in battery reversibility. In their Review, Peter Bruce and colleagues (article no. 16128) survey recent progress in understanding redox mechanisms at the oxygen electrode and their dependence on the electrolyte solutions. They also discuss ongoing challenges and possible solutions in maintaining the stability of electrodes and electrolytes during battery operation.

Lithium-sulfur, on the other hand, has its own intrinsic problems. The lithium polysulfide intermediates formed by the reduction of sulfur are soluble and can thus shuttle between the electrodes, which results in significant capacity loss and limits battery life. Linda Nazar and colleagues (article no. 16132) review recent advances in understanding the polysulfide chemistry in lithium-sulfur batteries, focusing on the use of various chemical interactions to trap polysulfides at the host electrode materials so as to mitigate their shuttling problem. They also discuss the key issues in developing multifunctional positive electrodes to achieve high sulfur loading, as well as multifunctional electrolytes to further regulate polysulfide dissolution.

Owing to the very high specific capacity of lithium, pure lithium metal would be the ideal negative electrode for lithium-ion batteries. Unfortunately, pure lithium is too reactive with liquid electrolytes; dendrite formation at the negative electrode, induced by the rough electro-deposition of lithium, can also pose a safety hazard. Such challenges have plagued the development of lithium-metal batteries for decades and only recently has research in this area gone through a renaissance. In their Perspective (article no. 16114), Lynden Archer and colleagues analyse the fundamentals of the electro-deposition in lithium-metal batteries and propose design principles for stable electrolytes and lithiummetal/electrolyte interfaces, which may offer guidelines for future development.

Meanwhile, solid-state batteries - which replace the conventional liquid electrolytes with solids with fast ion conductivity - offer an attractive alternative to lithium-ion for building safer batteries. Like many post lithium-ion technologies, both optimistic and pessimistic views exist regarding the prospects of solid-state batteries as a viable platform. Jürgen Janek and colleagues discuss in their
Comment (article no. 16141) a number of critical issues in realizing high-performing solid-state batteries, including cell mechanical integrity and long-term operation. They also offer their opinions on whether or not high voltage and high power density can be achieved and if the lithium-metal anode can bring the much needed capacity boost for future solid-state batteries.

The types of post lithium-ion battery presented in this Focus (www.nature.com/ post-lithium-ion-batteries) are by no means exhaustive. There are many other efforts in developing various battery technologies, each with their own advantages and drawbacks ${ }^{6}$. Sodium-ion, for example, benefits from the abundance of sodium sources and a similar electrochemistry to lithium-ion batteries, but sacrifices energy density because of sodium's greater ionic mass. Batteries based on multivalent ions, such as magnesium and aluminium, enable the transport of multiple charges, instead of the single electron offered by lithium-ion batteries. In so doing they create new problems such as low ion conductivity. Redox flow batteries have a completely different architecture from conventional lithium-ion, as the energy is stored in fuels flowing from external tanks in the form of two soluble redox couples. They have unique advantages in grid-scale storage, but often suffer from slow response, low durability and low energy efficiency. Of course, great challenges also exist in moving scientific discoveries through to commercial success, which brings a whole different dimension to battery development.

What we hope becomes apparent from this Focus is that breakthroughs at the fundamental level are needed to achieve forward leaps in performance - otherwise much battery development will still rely on slow and incremental optimizations. The advances in the fundamental understanding of battery processes highlighted here offer real hope for the realization of viable post lithium-ion batteries.

\footnotetext{
References

1. Crabtree, G., Kócs, E. \& Trahey, L. MRS Bulletin 40, 1067-1078 (2015)

2. Armstrong, R. C. et al. Nat. Energy 1, 15020 (2016).

3. Lithium ion NCR18650B specifications (Panasonic, 2012); http://go.nature.com/2b1p3ss

4. http://go.nature.com/2bloKaK

5. van Noorden, R. Nature 507, 26-28 (2014)

6. Choi, J. W. \& Aurbach, D. Nat. Rev. Mater. 1, 16013 (2016).
} 